\title{
Toward Father/Dad-Friendly Early Intervention for Families of Children with Special Educational Needs: A Qualitative Study
}

\author{
Ke Ren ${ }^{1 *}$, Conor Mc Guckin ${ }^{1}$ \\ 1. School of Education, Trinity College Dublin, Ireland
}

\begin{abstract}
.
While the participation of fathers/dads in the early intervention (EI) programme provided for families of children with special educational needs (SEN) has been proved to have a positive impact on the educational and experiential outcome of the child and the family, the level of fathers/dads involvement in EI continue to be low and the reasons for this are poorly understood. The purpose of this study was to explore fathers/dads' experiences of, and preferences for EI programme, as well as their perceptions of the barriers that limit their participation in EI. The roles and perceptions of EI professionals and mothers/mums in supporting the involvement of fathers/dads were also explored. Qualitative in-depth interviews were conducted with a total of 15 fathers/dads, EI professionals, and mothers/mums in Ireland. Findings revealed a significant gap in knowledge, views, and practice among fathers/dads, EI professionals, and mothers/mums towards the issue of father/dad involvement in EI. In addition, several barriers and preferences for father/dad-friendly EI programme were identified by participants. Implications for future efforts in developing such tailored EI services for fathers/dads are discussed.
\end{abstract}

Keywords: early intervention, father involvement, special educational needs; barriers; preferences

\section{Introduction}

Within family-centred early intervention (EI) principle, high-quality interactions that are empowering and enabling between service providers and families of children with special educational needs (SEN) have been found associated with optimal family outcomes (Dunst \& Trivette, 2009; Guralnick, 2005; McWilliam et al., 2010; Trivette et al., 2010). This requires a coordinated approach of partnership with both mothers and fathers, to ensure that every decision that was made is beneficial to the outcome of the child and the family. While mothers/mums are seen as the primary EI target and often exclusive participants in both EI service delivery and EI research, fathers/dads are noticeably absent from EI services and there has been very little research conducted to understand this phenomenon. This knowledge gap is strongly reflected in recent studies on parental involvement in EI, indicating that mothers/mums are the predominant research focus (Bagner, 2013; Bagner \& Eyberg, 2003; Curran, 2003; Zin \& Nor, 2017), yielding inconclusive findings about fathers/dads' need of support in EI and the impact of their participation in EI programmes. 


\section{$3^{\text {rd }}$ World Conference on Research in TEACHING and EDUCATION}

\section{3-25 April, 2021 Prague, Czech Republic}

Although research literature to date on fathers/dads role and involvement has mainly focused on fathering within the context of families of typically developing children, with the majority of evidence indicating that the involvement of fathers/dads during early years can have a positive impact on child outcomes in a variety of developmental domains and across different developmental stages (Paquette \& Dumont, 2013), it is believed that a similar shift in role responsibilities of fathers/dads and their involvement is expected in families of children with SEN/D. For example, the positive participation of fathers/dads contributes to the enduring relationships in families of children with SEN/D, lead to optimal child and family developmental outcomes (Zin \& Nor, 2017). In the context of EI, research has also found that directly involving fathers/dads in EI programmes not only promotes the effectiveness of intervention programmes within the context of a family and a child with SEN/D (Ingber \& Most, 2012; Justin Dyer et al., 2009; Zaidman-Zait et al., 2018), but also enhances fathers/dads' understanding of their role identity as individuals, lead to higher levels of engagement in EI-related activities, vocal communication, and parental sensitivity (Fox et al., 2015; Roopnarine \& Yildirim, 2019).

In view of the potential role that fathers/dads can play when their children and families receiving EI services, it is logical to expect greater father/dad participation in current family-centred EI practice. However, the recognition of fathers/dads role and their involvement in the lives of children with SEN/D and their families does not appear to be the case in family-centered EI. Mothers/mums continue to be seen as the primary EI target and agent for communication and voices and perceptions towards their involvement with EI services are not represented(Flippin \& Crais, 2011). Therefore, more knowledge regarding fathers/dads' expressed need for support and perceived barriers to, and preferences for EI service, as well as the adequacy of information support systems that are available to fathers/dads is needed. In addition, as some research has suggested that there is a lack of knowledge among EI professionals on how to provide appropriate and gender-sensitive service to fathers/dads of children with SEN/D(Flippin \& Crais, 2011; McBride et al., 2017; Raikes et al., 2005), it is essential to take EI professionals views towards fathers/dad involvement in EI in consideration. Furthermore, the core value of family-centered EI is to support the empowerment of both parents as active decision-makers so that they are able to advocate for their children's unique needs (Dunst et al., 1988; Fox et al., 2015). Thus, exploring the views and perceptions of mothers/mums towards father/dad involvement in EI is also significant as directly involving fathers/dads in EI services may ease the overall workload for mothers/mums, leads to reduced levels of stress and positive child and family outcome (Flippin \& Crais, 2011).

The purpose of this study was to take initial steps in influencing the area of EI to attend more fully on fathers/dads, as well as influence on the knowledge, attitudes, behaviour, and relationships amongst stakeholders/actors within the EI context in Ireland. Information gained from the qualitative data collected provides valuable insight into fathers/dads' experiences of, perceived barriers to, and preferences for EI services, the role, and perceptions of EI professionals in supporting and promoting the involvement of fathers/dads, as well as the wider perspective (mothers/mums perception) towards fathers/dads' involvement in the lives of children with SEN/D within the EI context. Such information is critical in supporting EI 


\section{$3^{\text {rd }}$ World Conference on Research in} TEACHING and EDUCATION

professionals to develop initiative services for fathers/dads who might be struggling in such parenting context.

\section{Method}

\subsection{Research Paradigm}

The purpose of the study and the interpretive philosophical stance underlying the study derived the design of a qualitative paradigm. The researcher sought answers for research by forming and underpinning diverse viewpoints of different individuals (fathers/dads, EI professionals, mothers/mums) from different groups(service providers, services users), in which it was very important when researching an area that is interdisciplinary in nature within the educational context. Furthermore, a lack of research in the area of father/dad's role and involvement within the EI context reinforced the use of a qualitative approach. It provided the researcher with opportunities to engage in dynamic interactions with research participants, to understand the research environment through self-interpretation, to construct multiple realities in a given context, and to finally translate the lived experience of participants into words and meanings that helps to inform the practice (Merriam, 2014). By using this inductive and inclusive approach, knowledge and intellectual progress on the area of EI and father/dad involvement was achieved.

\subsection{Participants}

A total of 15 fathers/dads ( $n=7)$, EI professionals $(n=3)$, and mothers/mums $(n=5)$ in Ireland participated in the one-to-one qualitative interview. All the participants were recruited via social media advertisements, or flyers distributed to three organisations that offered EI services and parenting support services to families of children with SEN. In terms of the participant selection criteria, fathers/dads (biological fathers and male caregivers) and mothers/mums (biological fathers and male caregivers) were eligible to participate in the study if they had a child with SEN and received EI services in Ireland. EI professionals who worked with families of children with SEN across all disciplines (e.g., therapeutic, early years, social work/care, family support, etc.) within the EI context in Ireland were eligible to participate.

\subsection{Data Collection and Analysis}

As the research was conducted during the time of the COVID -19 pandemic, a blended approach to data collection was applied. While five face-to-face interviews with fathers/dads $(n=2)$ and mothers/mums $(n=3)$ were conducted, all other participants $(n=10)$ of EI professionals $(n=3)$, fathers/dads $(n=5)$, and mothers/mums $(n=2)$ recruited participated in the one-to-one telephone interviews when the government lockdown and restrictions were imposed in Ireland. This unexpected change to the data collection method was beneficial. When interviewing fathers/dads of children with SEN about their perceptions of their involvement, role, and responsibilities, the telephone interview provided a more relaxed, less-threatening and 


\section{$3^{\text {rd }}$ World Conference on Research in} TEACHING and EDUCATION

personal, and comfortable platform for them to engage in in-depth conversations with the researcher.

The semi-structured interview contained open-ended questions, combined with a series of probing questions, which were designed and again guided by an extensive range of literature concerning father/dad's role and involvement within the EI context. All the interviews were recorded and transcribed verbatim. Each transcription was reviewed and analyzed using thematic analysis. To provide a more in-depth, intensive, and sharply focused exploration of issues pertaining to father/dad involvement in EI from different perspectives of each participant groups, as well as to understand relationships and links between these groups, a cross-case analysis was performed to explore commonalities and differences that emerged following the thematic analysis of each transcript from three different participant groups (EI professionals, fathers/dads, mothers/mums).

\section{Results}

Themes that emerged from the thematic analysis, followed by a cross-case analysis across three participant groups (EI professionals, fathers/dads, mothers/mums) were organised into: (1) perceptions on father/dad involvement in EI; (2) barriers and factors affect the involvement of fathers/dads; and (3) fathers/dads' preference and need for service.

\section{Theme 1. Perceptions on father/dad involvement in EI}

Fathers/dads' involvement contribute to family and child outcome

All the participants across three groups all believe that the involvement of fathers/dads contribute to family and child outcome. For fathers/dads, all of them believe that there is a need for them to be equally involved in the lives of their children with SEN: "you need to be there...it's a constant battle." For mothers/mums, the participation of their husbands/partners contributes to family and child outcome in many forms, such as EI-related decision making, long-term plan, reduce mothers/mums pressure, etc. All these contributions make "the whole family stand on the same battlefield, fighting for the same goal." For EI professionals, it was reported that the involvement of fathers/dads is very important in their work with families of children with SEN/D.

\section{Fathers/dads - the taboo topic in EI}

While all three groups have expressed a positive attitude towards father/dad involvement in EI, the finding also suggest that fathers/dads could be seen as a taboo topic in EI. Some of the fathers/dads interviewed believe that the professionals tend to avoid mentioning about fathers/dads as many families of children with SEN are father-absent, "they don't want to talk about fathers because a lot of time fathers are not in the picture." For mothers, the same feeling was reported, "you are on an awkward one...there's so many houses there that they don't have dads." When asked about EI professional's experience of working with fathers/dads, all three 


\section{$3^{\text {rd }}$ World Conference on Research in} TEACHING and EDUCATION

participants reported that they have to be cautious when talking about fathers/dads with families, to ensure that mothers/mums of single-parent families are not offended, "you have to word it very carefully."

\section{Theme 2. Barriers and factors affect the involvement of fathers/dads}

Fathers/dads' work or EI appointment?

All the EI professionals perceived fathers/dads' work as a major barrier to their involvement in EI. Such a barrier has directly contributed to the lack of presence of fathers/dads in EI appointments, activities, and parental training sessions, and therefore it is hard for EI professionals to engage with fathers/dads. This was reflected when asked about the most heard reason when fathers/dads are absent, one EI specialist said, "dad isn't there, he's at work." While many fathers/dads and mothers/mums also perceived fathers/dads' work as a major barrier to their participation in EI, however, they expressed more frustrations about the time of EI appointment. For example, when sharing their family's experience on attending EI appointments, the mother stated, "the time of the early intervention activities never matched my husband's time." Her husband also reported the same frustration, "I can't! They can choose their time alright!"

\section{Fathers' belief and attitude towards SEN and EI}

Fathers/dads' belief and attitude towards their children's SEN and EI was also indicated as a barrier to father/dad involvement, mainly by mothers/mums and EI professionals. All the EI professionals interviewed perceive the father/dad as being absent as they seem to be not interested in EI. One Autism intervention teacher stated, "they don't seem to get it," and an EI specialist said, "there seems to be a mistrust of outside help." For mothers/mums, fathers/dads' level of interest in EI and acceptance to their children's SEN was indicated as a barrier by all the participants. For example, a mother whose husband was quite involved stated, "I just think a lot fathers don't accept it." However, different perceptions were raised by fathers/dads when sharing their narrative of becoming a father/dad of a child with SEN. Whilst some participants did acknowledge that there are individual fathers/dads who do not accept their children's SEN, many reported the shocking, hurtful, and traumatic feelings when heard their child is diagnosed with a disability. One father emotionally said, "father is actually still dealing with trauma and the unexpected news," therefore they are not ready to go to face the professionals.

Gender roles regarding caregiving and help-seeking

Traditional gender roles regarding caregiving and help-seeking were mentioned by all three groups of participants. At a societal and cultural level, mothers/mums and EI professionals believe that fathers/dads perceive mothers/mums as the primary caregiver, just like one mother indicated, "just the man being the man." In terms of help-seeking, fathers/dads reported stigma associated with asking for help, as they viewed this as a sign of weakness or an indication that they were not coping. When commenting on this, one father explained, "men are funny, in some respects to be contradictory," and one social care worker said, "it can be a sense of bravado." 


\section{$3^{\text {rd }}$ World Conference on Research in TEACHING and EDUCATION}

23-25 April, 2021

Prague, Czech Republic

The gendered nature of EI service

It was very noticeable throughout all the interviews with all three groups of participants that EI service is perceived as a gendered service. This is indicated as a barrier that may affect the participation of fathers/dads. For example, many mothers/mums reported that due to the gendered nature of EI services where an all-female environment is created, fathers/dads often feel embarrassed and uncomfortable attending EI activities such as parental training programmes and workshops, "he felt uncomfortable and inferior being the only man sitting in the class." For fathers/dads, when asked if EI is predominantly focused on mothers/mums, many of them said, "EI doesn't tend to focus on mothers," but resignedly expressed, "that's just the way it is." For EI professionals, many believe that EI is mother-focused and EI professionals are female-dominated professions, "I have to accept that the reality... we are perceived as a female lead occupation."

\section{Theme 3. Fathers/dads' preference and need for service}

Activity-based intervention

Information and activities that were both relevant and interesting to fathers/dads were highlighted as a key preference by all three participant groups. While EI professionals reported that "there's more of a buy-in if there's a physical joining in," mothers/mums further explain it is because "this is a father's strengths." For fathers/dads themselves, a preference for interventions to be activity/event - based or run in social settings (e.g., a pub, outdoor park) was highlighted. This was strongly reflected when interviewing one father who is not very involved, where he said, "I might take the lead on that" if EI service can organise day out activities for all families of children with SEN.

\section{Father/dad-led support group}

Through our interviews with fathers/dads, a need for establishing father/dad-led peer support group was highlighted. Although fathers/dads acknowledged the benefits of having two parents attending EI appointments and being involved in support groups, they reported a need for having support groups established "for the fathers run by the fathers." Relatedly, fathers/dads also reported a preference for having fathers/dads who have experiences on parenting a child with SEN to run the support group, so that the relationships and trust amongst group members would be established. For mothers/mums and EI professionals, while mothers/mums feel there is a need to have "a separate time for the fathers to do his own thing," EI professionals believe they can "...get the father involved if there is a group."

\section{Father-dad targeted service}

All three groups believe the development of a father/dad-targeted service would encourage and promote the participation of fathers/dads in EI, as well as the lives of their children with SEN. Fathers/dads highlight that EI service should make a specific effort to target fathers/dads and 


\section{$3^{\text {rd }}$ World Conference on Research in TEACHING and EDUCATION}

make them feel welcomed; "fathers do need to feel that they can be involved." This includes addressing them on letters, information leaflets, workshop invitations, and recruitment and advertisement. This is supported by mothers/mums as such actions would "give them the option to show up." For EI professionals, while one said developing father/dad targeted service "it's just a matter of being mindful," other professionals believe it would be very hard to achieve in practice.

\section{Discussion}

While similarities on views and perceptions regarding fathers/dads' role in families of children with SEN and their involvement in and preferences for in EI was indicated in line with previous research, findings from our study also highlight a significant gap in knowledge, perceptions, and relationship among fathers/dads, EI professionals, and mothers/mums towards the issue of fathers/dads involvement in EI. Such disconnection in knowledge was mainly reflected in the perceived barriers to father/dad involvement as indicated by three groups of participants. Result from the cross-case analysis suggests whilst EI professionals perceive fathers/dads' lack of presence due to work as a barrier to their participation, both fathers/dads and mothers/mums also perceive the time and the form of EI appointment have a direct impact on fathers/dads participation. For us, this disconnection in knowledge is because fathers/dads' work and EI professionals' work all occur at the same time. Therefore, the issue of work must be addressed if we indeed want to encourage more fathers/dads to participate in EI services. Wider support from a policy perspective that allows fathers/dads of children with SEN to take extra time off work with full pay may address such issue. For EI services, financial incentives from the government are needed to encourage EI providers to provide evening and weekend services for families of children with SEN.

Another disconnection in knowledge that emerged from this study related to fathers/dads' beliefs and attitudes towards their children's SEN and EI. Both mothers/mums and EI professionals perceive this as a barrier to fathers/dads' involvement, with the views that many fathers/dads do not seem to accept their children's SEN. However, our findings from the fathers/dads themselves suggest this is not the case. Many fathers/dads expressed how deep they are emotionally affected by the diagnosis of a child's SEN and/or disability, and thus they are not ready to be fully involved in EI. This has been highlighted in previous research (Marsh et al., 2018) exploring fathers' experiences of parenting a child with a disability. Such finding indicates a need for EI services to develop post-diagnostic support for families, especially fathers/dads of children with SEN. Training for EI professionals in supporting fathers/dads' needs through this emotional upheaval is also important.

Through our study, the gendered nature of EI service was noticeably highlighted by fathers/dads, mothers/mums, and EI professionals as a barrier to father/dad involvement. Unlike previous study (Sicouri et al., 2018), where fathers reported a belief that EI services are predominantly mothers/mums-focused, both fathers/dads and mothers/mums in our study believe EI does not tend to focus on mothers/mums, rather, it just because mothers/mums are 
the ones who always attend EI appointment due to societal view on gender roles. Similarly, views were also expressed by EI professionals, highlighting that EI is a gendered service and they are perceived as a female-dominated occupation. Although such finding was consistently reported in other research (McBride et al., 2017), it is more noticeable in our research considering historically, Ireland had a strong tradition of gender segregation in education, labour, and parental role (Sheehan et al., 2017). It is possible that such traditional gender views still have a long-lasting impact on Irish society. Therefore, there is a need to implement strategies that raise societal awareness on father/dad involvement in the lives of young children with SEN/D and EI in general.

In summary, this study explored fathers/dads' experiences of, perceived barriers to, and preferences for EI services, the role, and perceptions of EI professionals in supporting and promoting the involvement of fathers/dads, as well as the wider perspective (mothers/mums perception) towards fathers/dads' involvement in the lives of children with SEN/D within the EI context. Such exploration enabled us to examine how stakeholders involved in such context were perceiving the issue of fathers/dads' role and involvement in EI, and thus to highlight the disconnections in knowledge, perceptions, and relationships among fathers/dads, mothers/mums, and EI professionals on the issue that was under investigation. The findings of this study have important implications for future efforts in developing more father/dad inclusive EI services from a policy and practice perspective.

\section{References}

Bagner, D. M. (2013). Father's role in parent training for children with developmental delay. Journal of Family Psychology, 27(4), 650-657. https://doi.org/10.1037/a0033465

Bagner, D. M., \& Eyberg, S. M. (2003). Father Involvement in Parent Training: When Does It Matter? Journal of Clinical Child and Adolescent Psychology, 32(4), 599-605. https://doi.org/10.1207/S15374424JCCP3204_13

Curran, L. (2003). Social Work and Fathers: Child Support and Fathering Programs. Social Work, 48(2), 219-227. https://doi.org/10.1093/sw/48.2.219

Dunst, C. J., \& Trivette, C. M. (2009). Meta-Analytic Structural Equation Modeling of the Influences of Family-Centered Care on Parent and Child Psychological Health. International Journal of Pediatrics, 1-10. https://doi.org/10.1155/2009/576840

Dunst, C. J., Trivette, C. M., Davis, M., \& Cornwell, J. (1988). Enabling and Empowering Families of Children With Health Impairments. Children's Health Care, 17(2), 71-81. https://doi.org/10.1207/s15326888chc1702_2

Flippin, M., \& Crais, E. R. (2011). The need for more effective father involvement in early autism intervention: A systematic review and recommendations. Journal of Early Intervention, 33(1), 24-50. https://doi.org/10.1177/1053815111400415 
Fox, G. L., Nordquist, V. M., Billen, R. M., \& Savoca, E. F. (2015). Father Involvement and Early Intervention: Effects of Empowerment and Father Role Identity. Family Relations, 64, 461-475. https://doi.org/10.1111/fare.12156

Guralnick, M. J. (2005). An overview of the developmental systems model for early intervention. In M. J. Guralnick (Ed.), The developmental systems approach to early intervention (pp. 3-28). Brookes.

Ingber, S., \& Most, T. (2012). Fathers' Involvement in Preschool Programs for Children With and Without Hearing Loss. American Annals of the Deaf, 157(3), 276-288. https://doi.org/10.1353/aad.2012.1620

Justin Dyer, W., McBride, B. A., Santos, R. M., \& Jeans, L. M. (2009). A longitudinal examination of father involvement with children with developmental delays: Does timing of diagnosis matter? Journal of Early Intervention, 31(3), 265-281. https://doi.org/10.1177/0192513X09340386

Marsh, L., Warren, P., \& Savage, E. (2018). “Something was wrong”: A narrative inquiry of becoming a father of a child with an intellectual disability in Ireland. British Journal of Learning Disabilities, 46(4), 216-224. https://doi.org/https://doi.org/10.1111/bld.12230

McBride, B. A., Curtiss, S. J., Uchima, K., Laxman, D. J., Santos, R. M., Weglarz-Ward, J., Dyer, W. J., Jeans, L. M., \& Kern, J. (2017). Father Involvement in Early Intervention: Exploring the Gap Between Service Providers' Perceptions and Practices. Journal of Early Intervention, 39(2), 71-87. https://doi.org/10.1177/1053815116686118

McWilliam, R. A., Casey, A. M., \& Sims, J. (2010). The Routines-Based Interview. Infants \& Young Children, 22(3), 224-233. https://doi.org/10.1097/iyc.0b013e3181abe1dd

Merriam, B. S. (2014). Qulitative Research: A guide to Design and Implementation, Revised and Expanded from Qualitative Research and Case Study Applications in Education. In Progress in Electromagnetics Research Symposium. Jossey Bass.

Paquette, D., \& Dumont, C. (2013). The Father-Child Activation Relationship, Sex Differences, and Attachment Disorganization in Toddlerhood. Child Development Research. https://doi.org/10.1155/2013/102860

Raikes, H., Summers, J., \& Roggman, L. (2005). Father Involvement in Early Head Start Programs. Fathering: A Journal of Theory, Research, and Practice about Men as Fathers, 3(1), 29-58. https://doi.org/10.3149/fth.0301.29

Sheehan, A., Berkery, E., \& Lichrou, M. (2017). Changing role of women in the Irish society: an overview of the female consumer. The Irish Journal of Management, 36(3), 162-171. https://doi.org/https://doi.org/10.1515/ijm-2017-0017

Sicouri, G., Tully, L., Collins, D., Burn, M., Sargeant, K., Frick, P., Anderson, V., Hawes, D., Kimonis, E., Moul, C., Lenroot, R., \& Dadds, M. (2018). Toward Father-friendly Parenting Interventions: A Qualitative Study. Australian and New Zealand Journal of Family Therapy, 39(2), 218-231. https://doi.org/10.1002/anzf.1307

Trivette, C. M., Dunst, C. J., \& Hamby, D. W. (2010). Influences of Family-Systems Intervention Practices on Parent-Child Interactions and Child Development. Topics in 
$3^{\text {rd }}$ World Conference on Research in TEACHING and EDUCATION
23-25 April, 2021

Prague, Czech Republic

Early Childhood Special Education, 30(1), 3-19.

https://doi.org/10.1177/0271121410364250

Zaidman-Zait, A., Most, T., Tarrasch, R., \& Haddad, E. (2018). Mothers' and fathers' involvement in intervention programs for deaf and hard of hearing children. Disability and Rehabilitation, 40(11), 1301-1309. https://doi.org/10.1080/09638288.2017.1297491

Zin, M. I. M., \& Nor, M. M. (2017). Father involvement, early intervention program and well-being of children with special needs. International Journal of Early Childhood Special Education, 9(1), 17-30. https://doi.org/10.20489/intjecse.330045 